\title{
Fatores associados às enteroparasitoses em crianças usuárias de creches comunitárias
}

\author{
Risk factors for enteroparasites in children attending community day care centers
}

\author{
Taize Cristina Fonsecaa, Fabrízio Furtado de Sousab, Fábio Peron Carballoc, \\ Alysson Rodrigo Fonsecad, Denise Maria Rover da Silva Rabelo ${ }^{e}$ \\ a Bióloga pela Universidade do Estado de Minas Gerais (UEMG). \\ b Agrônomo. Doutor em Engenharia Florestal. Professor e pesquisador da UEMG, Unidade Divinópolis. \\ c Educador Físico. Doutor em Educação. Professor e pesquisador da UEMG, Unidade Divinópolis. \\ d Agrônomo. Doutor em Ciências. Professor e pesquisador da UEMG, Unidade Divinópolis. \\ e Bióloga. Doutora em Ciências Biológicas. Professora e pesquisadora da UEMG, Unidade Divinópolis. \\ Fonte de Financiamento: FAPEMIC.
}

Resumo

Objetivo: Este trabalho teve como objetivo principal avaliar os fatores de risco associados à presença de enteroparasitoses em crianças usuárias de duas creches comunitárias do município de Divinópolis (MG).

Materiais e Métodos: Neste estudo prospectivo, exploratório e transversal, foram coletadas amostras de fezes de 86 crianças e estas foram analisadas através do método de Hoffman, Pons e Janer. As informações familiares e socioeconômicas das crianças foram obtidas através de um questionário. As condições higiênico-sanitárias das creches foram analisadas através de observação estruturada.

Resultados: A prevalência de enteroparasitoses encontrada foi de 15,1\%, sendo estas ocasionadas pelos protozoários Giardia lamblia (58,4\%) e Entamoeba coli (41,6\%), não sendo observada infecção por helmintos. Foram identificados alguns fatores de risco associados à presença de parasitoses intestinais, como a baixa escolaridade da mãe $(p=0,048)$, a presença de animais domésticos em casa $(p=0,028)$, o tempo de gestação inferior a 36 semanas $(p=0,022)$ e o tempo de amamentação inferior a seis meses $(p=0,009)$. As condições higiênico-sanitárias das creches foram consideradas adequadas.

Conclusão: Os resultados demonstram que características familiares estão relacionadas à ocorrência de enteroparasitoses em crianças e reforça a necessidade da educação/conscientização dos pais como fator essencial no controle dessas doenças.

Palavras-chave: fatores de risco; doença parasitária; criança.

Objective: The aim of this study was to evaluate the associated risk factors of enteroparasitosis in children from community day care centers maintained by the municipal government of Divinópolis (MG).

Materials and Methods: Prospective, exploratory and cross-sectional study. Eighty six fecal samples from children were analyzed by the Hoffman, Pons and Janer method. Family and socioeconomic information were evaluated by a questionnaire. The hygienic-sanitary aspects of community day care centers were analyzed through structured observation.

Results: The prevalence of enteroparasitosis found was 15.1\% and the main causes were protozoa Giardia lamblia (58.4\%) and Entamoeba coli (41.6\%). No infection by helminths were observed. Some risk factors associated to the presence of intestinal parasitoses were identified, including the mother's low education level $(p=0.048)$, the presence of domestic animal at home $(p=0.028)$, breastfeeding duration less than six months $(p=0.022)$ and gestational age less than 36 weeks $(p=0.009)$. The hygienicsanitary conditions of day care centers were considered adequate.

Conclusion: The results demonstrated that family characteristics are related to the occurrence of enteroparasitosis in children and reinforces the necessity of education/awareness of parents as an essential factor in the control of these diseases.

Keywords: risk factors; parasitic diseases; child. 


\section{INTRODUÇÃO}

As enteroparasitoses ainda representam um dos mais sérios problemas de saúde pública, tanto no Brasil ${ }^{1,2}$ como em outros países em desenvolvimento ${ }^{3}$. No Brasil, as helmintoses e protozooses apresentam alta prevalência, principalmente devido às condições climáticas do país, a presença de vetores mecânicos, a falta de políticas públicas efetivas e medidas de educação em saúde ${ }^{1}$.

Apesar das parasitoses intestinais acometerem indivíduos em qualquer faixa etária, a população infantil representa o grupo de maior susceptibilidade às infecções parasitárias, principalmente devido aos hábitos inadequados de higiene $^{2,3,5}$. Nesta faixa etária, as enteroparasitoses podem ocasionar diarreias, anemia e deficiência de nutrientes, comprometendo o desenvolvimento físico e cognitivo das crianças ${ }^{6}$.

Em virtude da maior participação feminina no mercado de trabalho, as creches passaram a ser o primeiro ambiente externo ao doméstico que a criança frequenta, tornando-se um potencial local de contaminação ${ }^{7}$. Estudos realizados têm demonstrado uma maior prevalência de parasitoses intestinais em crianças frequentadoras de creches, quando comparadas às crianças cuidadas em casa ${ }^{7}$. Isso provavelmente se deve à aglomeração e ao contato com outras pessoas, o que predispõem à transmissão de parasitas intestinais via contato direto, através da água ou alimentos contaminados ${ }^{8}$. Apesar disso, os fatores implicados na contaminação de crianças usuárias de creches por parasitas intestinais podem variar dependendo da região, o que demonstra a necessidade de estudos em regiões ainda não avaliadas. A partir da compreensão dos fatores que se relacionam à contaminação de crianças por enteroparasitas, será possível o investimento em medidas preventivas mais efetivas para a redução da prevalência de infecções enteroparasitárias.

Tendo em vista a escassez de trabalhos que avaliem esta temática, especialmente na cidade de Divinópolis-MG, o objetivo deste estudo foi verificar os principais fatores associados à ocorrência de parasitoses intestinais em crianças, com idade entre seis meses e três anos, usuárias de creches comunitárias do município de Divinópolis-MG.

\section{MATERIAIS E MÉTODOS}

Trata-se de um estudo analítico, transversal, individual, não controlado. O estudo foi realizado no município de Divinópolis, localizado na região centro oeste do estado de Minas Gerais $\left(20^{\circ} 8^{\prime} 21^{\prime \prime}\right.$ de latitude sul e $44^{\circ} 53^{\prime} 17^{\prime \prime}$ de longitude oeste). O município apresenta uma extensão territorial de 716 quilômetros quadrados e sua população é de aproximadamente 216 mil habitantes 9 . Divinópolis apresenta um dos melhores índices de saneamento de Minas Gerais, sendo que 95,4\% dos moradores possuem água encanada e tratada, 81,4\% possuem instalações sanitárias com rede geral de esgoto e 93,7\% das casas dispõem de coleta de lixo, segundo o IBGE (2010) ${ }^{9}$.

O município de Divinópolis possui quatro creches comunitárias e dentre estas, duas concordaram com a realização do estudo. Cada uma destas creches acolhe cerca de 50 crianças, com faixa etária entre seis meses e três anos, sendo que uma está localizada na região central e outra na região periférica do município.

Foram incluídas no estudo todas as crianças, com idade entre seis meses e três anos, de ambos os sexos, cujos pais ou responsáveis concordaram com a pesquisa e assinaram o Termo de Consentimento Livre e Esclarecido. Todas as atividades desenvolvidas neste trabalho foram fundamentadas e respaldadas pela Resolução do Conselho Nacional de Saúde no 466, de 2012 (CAAE 09057612.3.0000.5115).

Para analisar as condições higiênico-sanitárias das creches foi elaborado um roteiro estruturado de observação, tendo como base o roteiro de inspeção sanitária em creches desenvolvido por Silva e Matté ${ }^{10}$. Dentre os itens contidos no roteiro, foram selecionados para compor o roteiro de observação deste estudo aqueles que tinham relação com as condições higiênico-sanitárias das instituições, como: forma de abastecimento de água, presença de rede de esgoto, presença de instalações sanitárias para as crianças, existência de solário, existência de refeitório com locais separados para recepção, lavagem e preparo das mamadeiras, esterilização e distribuição de materiais, forma de condicionamento do lixo e cuidados no manuseio dos alimentos.

A observação foi realizada por um único pesquisador, em três dias diferentes. No preenchimento do roteiro, o pesquisador classificou os itens como adequados ou inadequados. Na análise final, o item foi considerado adequado quando obtinha essa avaliação por, no mínimo, duas vezes.

Para verificar a presença de parasitas intestinais, foram coletadas três amostras de fezes de cada criança. As coletas das amostras de fezes foram feitas nas creches, pelas monitoras responsáveis pelas crianças, durante o mês de abril de 2014. As amostras coletadas foram armazenadas em um recipiente devidamente etiquetado, contendo uma solução conservante de Merthiolate, lodo e Formol (MIF). O Material coletado foi encaminhado a um laboratório particular de análises clínicas.

Para avaliar a existência de parasitas, as amostras de fezes foram analisadas através do método de sedimentação ${ }^{11}$. O material coletado foi transferido para um béquer e dissolvido em água destilada, com o auxílio de um bastão de vidro. A suspensão formada foi filtrada através de tela de náilon, 
em um cálice de sedimentação de $200 \mathrm{~mL}$ de capacidade. Os detritos retidos foram lavados com água destilada, sendo que esta foi recolhida no mesmo cálice. Posteriormente, o volume do cálice foi completado com mais água destilada e colocado para sedimentar por duas a quatro horas ou até que ocorresse a formação de sedimento. Após este período, o líquido sobrenadante turvo foi descartado e o volume do cálice completado novamente com água destilada para nova sedimentação. Quando o líquido sobrenadante encontrouse límpido, uma parte do mesmo foi descartado e uma amostra de sedimento do vértice do cálice de sedimentação foi coletada. Esta amostra foi colocada sobre quatro lâminas, que foram analisadas através de microscopia óptica comum, com aumentos de 10 e 40 vezes, sem a adição de corante.

Os demais dados foram obtidos através de um questionário fechado encaminhado aos pais das crianças participantes. Foram investigados como fatores de risco associados às enteroparasitoses: o nível socioeconômico familiar (renda familiar, número de filhos); as condições sanitárias das moradias (formas de abastecimento de água e presença de rede de esgoto), as características da mãe das crianças (idade, escolaridade, estado civil), além das características da criança (idade, sexo, raça, peso ao nascer, tempo de amamentação, tipo de leite utilizado, tempo de gestação, frequência na realização de exames de rotina e de fezes, uso de chupeta e mamadeira) e presença de animais domésticos em casa.

Com relação às variáveis testadas, a raça das crianças pôde ser declarada como branca, parda ou negra, sendo as duas últimas agrupadas para análise. Para classificação do peso ao nascer, utilizou-se o critério da Organização Mundial da Saúde (OMS): baixo peso (crianças com menos de 2500g), peso insuficiente (2500g a $2999 \mathrm{~g}$ ) peso adequado (3000g a $3999 \mathrm{~g}$ ) e excesso de peso (4000g ou mais) ${ }^{12}$, sendo os dois primeiros agrupados para análise. Com relação ao tempo de gestação, as crianças que nasceram com idade gestacional inferior a 37 semanas foram consideradas prematuras ${ }^{12}$.

Os dados obtidos foram armazenados em um programa de banco de dados e analisados utilizando o software SPSS 20.0. Na estatística descritiva, utilizou-se as frequências absolutas e relativas (\%) das variáveis de maior relevância para a construção de tabelas. Para avaliar a relação entre os dados obtidos, foi realizado o teste $\chi^{2}$ - (qui-quadrado), sendo considerados significativos os valores de $p \leq 0,05$.

\section{RESULTADOS}

Foram avaliadas 86 crianças, sendo 40 meninas e 46 meninos (Tabela 1). A prevalência de enteroparasitoses foi de $15,1 \%$ (13 crianças) e todas as infecções observadas foram causadas por protozoários, sendo identificados Giardia lamblia e Entamoeba coli, com frequência de 58,4\% e 41,6\%, respectivamente. Não foi observado nenhum caso de bi ou poliparasitismo.

Tabela 1. Descrição da amostra das crianças por sexo, de acordo com a presença de parasitas e a idade das crianças.

\begin{tabular}{lcccccc}
\hline \multirow{2}{*}{ Variável } & \multicolumn{2}{c}{ Geral } & \multicolumn{2}{c}{ Feminino } & \multicolumn{2}{c}{ Masculino } \\
\cline { 2 - 7 } & $\mathbf{n}$ & $\%$ & $\mathbf{n}$ & $\%$ & $\mathbf{n}$ & $\%$ \\
Presença de parasitas & & & & & & \\
$\quad$ Sim & 13 & 15,1 & 6 & 15,0 & 7 & 15,2 \\
$\quad$ Não & 73 & 84,9 & 34 & 85,0 & 39 & 84,8 \\
Idade & & & & & & \\
0 a 1 ano & 10 & 11,6 & 4 & 10,0 & 6 & 13,0 \\
1 a 2 anos & 34 & 39,5 & 15 & 37,5 & 19 & 41,3 \\
2 a 3 anos & 42 & 48,8 & 21 & 52,5 & 21 & 45,7 \\
Total & 86 & & 40 & & 46 & \\
\hline
\end{tabular}

Não foi encontrada relação significativa entre a presença de parasitas intestinais e o sexo, a raça ou a idade das crianças avaliadas. Da mesma forma, não houve diferença significativa na prevalência de enteroparasitoses quando analisadas as creches separadamente. Sendo assim, os resultados foram analisados considerando como amostra do estudo o grupo de crianças pertencentes às duas creches (Tabela 2).

Dentre os fatores avaliados, o fato da criança residir apenas com a mãe não apresentou relação significativa com a presença de infecção por parasitas intestinais. No entanto, a escolaridade da mãe mostrou-se significativamente associada à presença de enteroparasitas nas crianças. A maior parte das mães das crianças parasitadas não concluiu o ensino médio $(69,2 \%)(p=0,048)$. Os demais fatores relacionados à mãe das crianças não apresentaram relação com a presença de enteroparasitoses, como a idade, o estado civil, o número de filhos e a renda familiar (Tabela 2).

Com relação às residências das crianças analisadas, $100 \%$ apresentam fornecimento de água por rede pública de abastecimento e coleta de lixo pelo sistema público de limpeza. Existe esgotamento sanitário em 69,2\% das casas e nas demais existem fossas sépticas (30,8\%). Os fatores de risco epidemiológicos analisados nas residências das crianças, como a forma de abastecimento de água na residência e a presença de rede de esgoto, não demonstraram diferença significativa quando confrontados à presença de positividade para enteroparasitas.

Por outro lado, a presença de animais de estimação nas residências apresentou associação significativa com a presença de infecção por parasitas intestinais. A maioria das crianças parasitadas convive com animais de estimação em casa $(76,9 \%)(p=0,028)$, sendo estes, em todos os casos, cães (Tabela 2). 
Tabela 2. Análise bivariada dos fatores relacionados à presença de parasitose em crianças, com idade entre 0 e 3 anos, das creches comunitárias analisadas.

\begin{tabular}{|c|c|c|c|c|c|c|}
\hline \multirow{2}{*}{ Variáveis } & \multicolumn{2}{|c|}{ Parasitária } & \multicolumn{2}{|c|}{ Não parasitária } & \multirow{2}{*}{ Total } & \multirow{2}{*}{ Valor de } \\
\hline & $\mathbf{n}$ & $\%$ & $\mathbf{n}$ & $\%$ & & \\
\hline \multicolumn{7}{|l|}{ Sexo } \\
\hline Masculino & 7 & 15,2 & 39 & 84,8 & 46 & 0,978 \\
\hline Feminino & 6 & 15,0 & 34 & 85,0 & 40 & \\
\hline \multicolumn{7}{|l|}{ Idade } \\
\hline De 0 a 1 ano & 1 & 10,0 & 9 & 90,0 & 10 & 0,604 \\
\hline De 1 a 2 anos & 4 & 11,8 & 30 & 88,2 & 34 & \\
\hline De 2 a 3 anos & 8 & 19,0 & 34 & 81,0 & 42 & \\
\hline \multicolumn{7}{|l|}{ Creche } \\
\hline Creche A & 5 & 10,9 & 41 & 89,1 & 46 & 0,238 \\
\hline Creche B & 8 & 20,0 & 32 & 80,0 & 40 & \\
\hline \multicolumn{7}{|l|}{ Cor/raça } \\
\hline Branca & 5 & 13,2 & 33 & 86,8 & 38 & 0,652 \\
\hline Não branca & 8 & 16,7 & 40 & 83,3 & 48 & \\
\hline \multicolumn{7}{|l|}{ Com quem a criança mora } \\
\hline Apenas com a mãe & 8 & 23,5 & 26 & 76,5 & 34 & 0,057 \\
\hline Com a mãe e com o pai & 4 & 8,0 & 46 & 92,0 & 50 & \\
\hline Sem nenhum dos pais & 1 & 50,0 & 1 & 50,0 & 2 & \\
\hline \multicolumn{7}{|l|}{ Escolaridade da mãe } \\
\hline Fundamental incompleto & 2 & 14,3 & 12 & 85,7 & 14 & 0,048 \\
\hline Fundamental completo & 1 & 9,1 & 10 & 90,9 & 11 & \\
\hline Médio incompleto & 6 & 40,0 & 9 & 60,0 & 15 & \\
\hline Médio completo & 3 & 7,3 & 38 & 92,7 & 41 & \\
\hline Superior & 1 & 20,0 & 4 & 80,0 & 5 & \\
\hline \multicolumn{7}{|l|}{ Idade da mãe } \\
\hline Menos que 20 anos & 0 & 0,0 & 3 & 100,0 & 3 & 0,728 \\
\hline De 20 a 25 anos & 4 & 16,0 & 21 & 84,0 & 25 & \\
\hline De 25 a 30 anos & 6 & 21,4 & 22 & 78,6 & 28 & \\
\hline De 30 a 35 anos & 1 & 9,1 & 10 & 90,9 & 11 & \\
\hline Acima de 35 anos & 2 & 10,5 & 17 & 89,5 & 19 & \\
\hline \multicolumn{7}{|l|}{ Estado civil da mãe } \\
\hline Solteira & 8 & 21,1 & 30 & 78,9 & 38 & 0,204 \\
\hline Casada/União consensual/União estável & 4 & 8,9 & 41 & 91,1 & 45 & \\
\hline Separada/Divorciada & 1 & 33,3 & 2 & 66,7 & 3 & \\
\hline \multicolumn{7}{|l|}{ Número de filhos } \\
\hline Um & 8 & 16,7 & 40 & 83,3 & 48 & 0,645 \\
\hline Dois & 2 & 9,1 & 20 & 90,9 & 22 & \\
\hline Três ou mais & 3 & 18,8 & 13 & 81,3 & 16 & \\
\hline \multicolumn{7}{|l|}{ Renda familiar } \\
\hline Até $\mathrm{R} \$ 545,00$ & 1 & 7,1 & 13 & 92,9 & 14 & 0,476 \\
\hline Entre $\mathrm{R} \$ 545,00$ e $1.200,00$ & 11 & 19,3 & 46 & 80,7 & 57 & \\
\hline Entre $R \$ 1.200,00$ e $1.800,00$ & 1 & 9,1 & 10 & 90,9 & 11 & \\
\hline Acima de $\mathrm{R} \$ 1.800,00$ & 0 & 0,0 & 4 & 100,0 & 4 & \\
\hline \multicolumn{7}{|l|}{ Abastecimento de água na residência } \\
\hline Poço ou cisterna & 0 & 0,0 & 2 & 100,0 & 2 & 0,546 \\
\hline Rede pública & 13 & 15,5 & 71 & 84,5 & 84 & \\
\hline \multicolumn{7}{|l|}{ Rede de esgoto na residência } \\
\hline Existe & 9 & 13,6 & 57 & 84,6 & 66 & 0,486 \\
\hline Não existe & 4 & 20,0 & 16 & 80,0 & 20 & \\
\hline \multicolumn{7}{|l|}{ Animal de estimação } \\
\hline Possui & 10 & 23,8 & 32 & 76,2 & 42 & 0,028 \\
\hline Não possui & 3 & 6,8 & 41 & 93,2 & 44 & \\
\hline
\end{tabular}


Com relação às características das crianças analisadas, o uso de chupeta ou mamadeira não apresentou relação com a existência de enteroparasitose (Tabela 3). Da mesma forma, não foi observada relação entre a presença de parasitismo intestinal e o peso da criança ao nascer, o fato de realizar exames periódicos e o tipo de leite fornecido à criança.

No entanto, o tempo de amamentação materna e o tempo de gestação apresentaram relação significativa com a presença de parasitas intestinais. Crianças amamentadas com leite materno por um período de tempo superior a seis meses tiveram menor prevalência de enteroparasitoses $(2,8 \%)$ que crianças amamentadas por até seis meses ou menos $(24,0 \%)(p=0,022)$. Com relação ao tempo de gestação, os dados demonstraram que crianças oriundas de uma gestação com duração superior a 37 semanas apresentaram menor prevalência de parasitoses intestinais $(9,1 \%)$ quando comparados às crianças provenientes de uma gestação com duração inferior a 37 semanas $(35,0 \%)(p=0,009)$ (Tabela 3).

As observações feitas nas creches durante as visitas permitiram constatar que ambas as creches possuem boa infraestrutura física e profissionais atenciosos. Nas duas creches, as cozinheiras usam avental, touca e luvas; as mamadeiras são lavadas em água corrente e posteriormente fervidas. As crianças possuem toalhas, escovas de dente e banheiros individuais; tomam dois banhos por dia e passam a maior parte do tempo em brincadeiras/atividades ao ar livre (ambas as creches possuem quadras). As fraldas usadas são descartadas em lixeiras com tampas, exclusivas para o descarte das mesmas. As duas creches estão localizadas em ruas asfaltadas, recebem água tratada, possuem rede de esgoto e caixa d'água própria. Em ambas as creches, a coleta de lixo ocorre três vezes por semana.

Tabela 3. Análise bivariada dos fatores relacionados à presença de parasitose em crianças, com idade entre 0 e 3 anos, das creches comunitárias analisadas.

\begin{tabular}{|c|c|c|c|c|c|c|}
\hline \multirow{2}{*}{ Variáveis } & \multicolumn{2}{|c|}{ Parasitária } & \multicolumn{2}{|c|}{ Não parasitária } & \multirow{2}{*}{ Total } & \multirow{2}{*}{ Valor de $p$} \\
\hline & $\mathrm{n}$ & $\%$ & $\mathrm{n}$ & $\%$ & & \\
\hline \multicolumn{7}{|l|}{ Chupeta } \\
\hline Usa & 9 & 23,1 & 30 & 76,9 & 39 & 0,154 \\
\hline Já usou & 2 & 11,8 & 15 & 88,2 & 17 & \\
\hline Nunca usou & 2 & 6,7 & 28 & 93,3 & 30 & \\
\hline \multicolumn{7}{|l|}{ Mamadeira } \\
\hline Usa & 9 & 13,8 & 56 & 86,2 & 65 & 0,619 \\
\hline Já usou & 4 & 21,1 & 15 & 78,9 & 19 & \\
\hline Nunca usou & 0 & 0,0 & 2 & 100,0 & 2 & \\
\hline \multicolumn{7}{|l|}{ Tempo de gestação } \\
\hline Inferior a 37 semanas & 7 & 35,0 & 13 & 65,0 & 20 & 0,010 \\
\hline Igual ou superior a 37 semanas & 6 & 9,1 & 60 & 90,9 & 66 & \\
\hline \multicolumn{7}{|l|}{ Tempo amamentação } \\
\hline Não amamentou & 1 & 16,7 & 5 & 83,3 & 6 & 0,022 \\
\hline Inferior a 6 meses & 11 & 25,0 & 33 & 75,0 & 44 & \\
\hline Por pelo menos 6 meses ou mais & 1 & 2,8 & 35 & 97,2 & 36 & \\
\hline \multicolumn{7}{|l|}{ Peso ao nascer } \\
\hline Baixo & 2 & 16,7 & 10 & 83,3 & 12 & 0,827 \\
\hline Adequado & 11 & 15,3 & 61 & 84,7 & 72 & \\
\hline Excesso & 0 & 0,0 & 2 & 100,0 & 2 & \\
\hline \multicolumn{7}{|l|}{ Leite é fornecido à criança } \\
\hline Leite em pó tipo NAN & 4 & 18,2 & 18 & 81,8 & 22 & 0,704 \\
\hline Leite de soja & 1 & 33,3 & 2 & 66,7 & 3 & \\
\hline Leite de vaca & 8 & 13,6 & 51 & 86,4 & 59 & \\
\hline A criança não toma leite & 0 & 0,0 & 2 & 100,0 & 2 & \\
\hline \multicolumn{7}{|l|}{ Realização de exames de rotina } \\
\hline Sim & 6 & 17,1 & 29 & 82,9 & 35 & 0,720 \\
\hline Somente quando está doente & 7 & 14,6 & 41 & 85,4 & 48 & \\
\hline Não & 0 & 0,0 & 3 & 100,0 & 3 & \\
\hline \multicolumn{7}{|l|}{ Exame de fezes } \\
\hline Já realizou & 6 & 16,2 & 31 & 83,8 & 37 & 0,805 \\
\hline Nunca realizou & 7 & 14,3 & 42 & 85,7 & 49 & \\
\hline
\end{tabular}




\section{DISCUSSÃO}

Neste estudo, a prevalência de infecção por parasitas intestinais foi menor do que a encontrada em estudos realizados em outras regióes brasileiras, com crianças na mesma faixa etária e com procedimentos metodológicos similares ao utilizado ${ }^{2,7,13}$. Apesar de descrito na literatura que crianças usuárias de creches apresentam maiores predisposições para o desenvolvimento de infecções por enteroparasitas ${ }^{7}$, é possível que a baixa prevalência de enteroparasitoses encontrada em nosso estudo esteja relacionada às boas condições higiênico-sanitárias observadas nas creches e às adequadas condições de saneamento relatadas como existentes nas residências das crianças. Este dado reforça a importância do saneamento básico adequado na prevenção das parasitoses intestinais ${ }^{14}$.

Dentre as crianças analisadas, não foi observada infecção por helmintos, somente por protozoários, sendo mais frequente a infecção por Giardia lamblia. Outros estudos realizados no Brasil também apontam a giardíase como a principal enteroparasitose observada em crianças ${ }^{5,15,16}$. A contaminação por Giardia lamblia ocorre mais frequentemente através da ingestão de cistos, contidos em água sem tratamento, alimentos contaminados, através de pessoa para pessoa, veiculados por vetores ou ainda através do contato com animais infectados ${ }^{16}$. Essas formas de transmissão facilitam a disseminação da giardíase, especialmente em locais com aglomeração de pessoas, como é o caso das creches.

Dentre os fatores avaliados, a presença de cães no ambiente doméstico apresentou relação significativa com a presença de parasitas intestinais nas crianças analisadas. Este dado corrobora com outros estudos que demonstram que o convívio com animais de estimação constitui um fator de risco para a contaminação por parasitas ${ }^{17,18,19}$. No caso específico da giardíase, infecção mais frequente observada neste estudo, Almeida e colaboradores (2010) demonstraram que crianças que convivem com cães parasitados pelo protozoário Giardia lamblia apresentam maiores chances de desenvolver a doença ${ }^{19}$. A contaminação animal-pessoa é favorecida quando o animal pode transitar livremente pelas ruas, ficando sujeito à contaminação por parasitas através da ingestão de água e alimentos contaminados, existindo também a possibilidade de transportar cistos de parasitas através dos pêlos ${ }^{17}$. Sendo assim, cães e outros animais de estimação devem ser rotineiramente avaliados por exames parasitológicos e tratados para eliminar a possibilidade de infectar outros animais ou membros da família'18,19.

Reforçando os dados já descritos na literatura, neste estudo não foi observada diferença significativa na prevalência de enteroparasitoses entre os gêneros ${ }^{5,15}$. Na faixa etária analisada neste estudo, de 6 meses a 3 anos de idade, meninos e meninas apresentam poucas diferenças de desenvolvimento neuropsicomotor relacionadas ao gênero ${ }^{19}$, o que sugere que crianças de ambos os sexos, nesta faixa etária, apresentam o mesmo nível de exposição ambiental.

A fase da infância é a que apresenta maior prevalência de enteroparasitoses devido, principalmente, aos hábitos higiênicos precários das crianças, à ausência de imunidade às infecções e reinfecções e à dependência de cuidados alheios ${ }^{16}$. Sendo assim, é de fundamental importância o conhecimento dos pais acerca das formas de contaminação e dos riscos das infecções por parasitas intestinais. Segundo estudos realizados, o cuidado dos pais para com as crianças menores e o grau de instrução dos mesmos pode diminuir o risco de contaminação por enteroparasitas ${ }^{21,22}$.

Corroborando com este fato, uma característica materna que mostrou relação significativa com a presença de parasitismo intestinal nas crianças foi a escolaridade. Crianças filhas de mães com menor escolaridade apresentaram maior prevalência de enteroparasitoses quando comparadas às crianças filhas de mães de maior escolaridade. Outros trabalhos na literatura também apresentaram esta relação entre escolaridade dos pais e presença de parasitas intestinais em crianças $5,13,23$, o que reforça a importância da instrução dos pais na prevenção das contaminações enteroparasitárias.

A educação dos pais tem favorecido a disseminação de hábitos e práticas fundamentais para o desenvolvimento das crianças, como é o caso do aleitamento materno. A importância do aleitamento materno é comprovada por diversos estudos científicos ${ }^{24,25}$ e dentre os vários benefícios do aleitamento materno pode ser citado a redução dos riscos de infecções bacterianas e virais ${ }^{26}$. Em nosso estudo foi observado que crianças que receberam amamentação materna por mais de seis meses tiveram uma menor prevalência de infecções parasitárias quando comparadas às crianças que amamentaram por até seis meses ou menos. Segundo Cacho \& Lawrence ${ }^{27}$, o leite materno protege as crianças contra infecções devido ao fato de apresentar compostos com ação antimicrobiana, anti-inflamatória, imunomoduladora, além de leucócitos. Devido à imaturidade do sistema imune, o recém-nascido é mais vulnerável às infecções, sendo fundamental a proteção conferida pela amamentação ${ }^{28}$. Substâncias imunológicas e fatores de crescimento presente no colostro e no leite materno protegem a mucosa intestinal contra a invasão de patógenos ${ }^{24,27,28}$ e portanto, reduzem os riscos de infecção enteroparasitária.

Outro fator que mostrou relação significativa com a presença de enteroparasitas nas crianças analisadas foi 
o tempo de gestação. É sabido que o nascimento prétermo pode acarretar prejuízos à saúde das crianças, como hemorragia intraventricular, hipóxia neonatal e outras intercorrências neurológicas que, de acordo com sua intensidade e duração, podem resultar em sequelas permanentes que vão interferir em diferentes aspectos da vida da criança ${ }^{29}$. Além desses riscos, por razões ainda não elucidadas, neste estudo foi observado que crianças oriundas de uma gestação com duração inferior a 37 semanas apresentaram maior prevalência de enteroparasitoses do que crianças nascidas a termo.

Este é o primeiro trabalho que descreve uma associação entre tempo de gestação e infecção por parasitas intestinais em crianças. A descrição desta correlação é de grande importância e sugere que as infecções enteroparasitárias devem ser mais uma preocupação entre os pais e pediatras de crianças nascidas prematuras. Da mesma forma, a correlação entre tempo de gestação e presença de enteroparasitoses reforça a importância do acompanhamento pré-natal, que deve iniciar no momento em que a gravidez é confirmada, a fim de manter a integridade das condições de saúde da mãe e do bebê.

Devido ao fato deste estudo apresentar limitações relacionadas à sensibilidade do método de diagnóstico, a possibilidade de erros relativos ao instrumento utilizado na obtenção dos dados sociodemográficos e familiares, bem como ao tamanho da população amostrada, sugere-se que outros estudos analisem também a relação entre tempo gestacional e a presença de enteroparasitas em crianças, para que a mesma possa ser confirmada.

Atualmente, a permanência de crianças pequenas em creches tem se tornado uma realidade para muitas famílias, e esta instituição muito tem contribuído para o desenvolvimento das crianças, tanto em seus aspectos físico, psicológico, intelectual, como também social ${ }^{15}$. Porém, devido ao contato frequente com outras crianças e adultos, as crianças usuárias de creches acabam mais expostas aos agentes infecciosos, ficando mais suscetíveis às doenças, inclusive às parasitoses intestinais ${ }^{7,15}$.

Apesar disso, neste trabalho foi observado que características familiares e das próprias crianças apresentaram associação significativa com a presença de infecção por enteroparasitas. Como as crianças na idade amostrada ainda não apresentam consciência das formas e dos riscos de contaminação por enteroparasitas, o papel dos pais neste cuidado é de fundamental importância. Sendo assim, para que haja um controle mais efetivo das enteroparasitoses, a conscientização dos pais acerca dessas doenças é uma estratégia de prevenção a ser adotada. Para isso, verifica-se a necessidade de ampliação de programas que visem à conscientização dos pais acerca das enteroparasitoses, como importante medida de prevenção dessas doenças.

\section{AGRADECIMENTOS}

Ao Programa Institucional de bolsas de iniciação científica - PIBIC/FAPEMIG/UEMG e ao Laboratório Centro de Diagnóstico de Divinópolis, Minas Gerais.

\section{REFERÊNCIAS}

1. Andrade EC, Leite ICG, Rodrigues VO, Cesca MG. Parasitoses intestinais: uma revisão sobre seus aspectos sociais, epidemiológicos, clínicos e terapêuticos. Rev APS. 2010;13:231-40.

2. Ferreira GR, Andrade CFS. Alguns aspectos socioeconômicos relacionados a parasitoses intestinais e avaliação de uma intervenção educativa em escolares de Estiva Gerbi, SP. Rev Soc Bras Med Trop. 2005;38(5):402-5. https://doi.org/10.1590/S003786822005000500008

3. Gamboa MI, Giambelluca LA, Navone GT. Spatial distribution of intestinal parasites in the City of La Plata, Argentina. Medicina. 2013;74(5):363-70.

4. Costa SSD, Silva BFP, Morais AFC, Wanderley FS. Ocorrência de parasitas intestinais em material subungueal e fecal em crianças de uma creche no município de Maceió-Alagoas. Pediatria. 2009;31(3):198-203.

5. Carvalho TBD, Carvalho LRD, Mascarini LM. Occurrence of enteroparasites in day care centers in Botucatu (São Paulo State, Brazil) with emphasis on Cryptosporidium sp., Giardia duodenalis and Enterobius vermicularis. Rev Inst Med Trop São Paulo. 2006;48(5):269-73. https://doi.org/10.1590/S003646652006000500006

6. Bethony J, Brooker S, Albonico M, Geiger SM, Loukas A, Diemert D, Hotez PJ. Soil-transmitted helminth infections: ascariasis, trichuriasis, and hookworm. Lancet. 2006;367(9521):1521-32. https://doi. org/10.1016/S0140-6736(06)68653-4

7. Gurgel RQ, Cardoso GDS, Silva AM, Santos LND, Oliveira RCVD. Creche: ambiente expositor ou protetor nas infestações por parasitas intestinais em Aracaju, SE. Rev Soc Bras Med Trop. 2005;38(3): 267-9. https://doi.org/10.1590/S0037-86822005000300014

8. Takayanagui OM, Capuano DM, Oliveira CA, Bergamini AM, Okino MH, Castro e Silva AA, Takayanagui AM. Analysis of the vegele productive chain in Ribeirão Preto, SP. Rev Soc Bras Med Trop. 2006;39(2):224-6. https://doi.org/10.1590/S003786822006000200018

9. Instituto Brasileiro de Geografia E Estatística (IBGE). Censo demográfico [Internet]; 2010 [capturado 2012 Jan 07]. Disponível em: http://www.ibge.gov.br/home/estatistica/populacao/censo2010/ default.shtm

10. Silva VAE, Matté $M H$. Inspeção sanitária em creches: uma proposta de roteiro de inspeção. Rev Direito Sanit. 2009;10(2):29-63. https:// doi.org/10.11606/issn.2316-9044.v10i2p29-63

11. Hoffman WA, Pons JA, Janer JL. The sedimentation-concentration method in schistosomiasis mansoni. PR J Public Health Trop Med.1934;9(3):283-9. 
12. Brasil. Ministério da Saúde. Secretaria de Políticas Públicas. Saúde da mulher. Assistência pré-natal: manual técnico [Internet]. Brasília (DF): Ministério da Saúde; 2000 [capturado 2017 Fev 02]. Disponível em: http://bvsms.saude.gov.br/bvs/publicacoes/cd04_11.pdf

13. Mascarini LL, Donalísio-Cordeiro MR. Helmintíases em crianças institucionalizadas em creches no município de Botucatu/SP, Brasil. Rev Patol Trop. 2007;36(2):149-58. https://doi.org/10.5216/rpt. v36i2.1788

14. Alves de Oliveira Serra MA, Chaves CS, Branco Coêlho ZC, Castro Rodrigues NL, Martins Vale J, Teixeira MJ, Oliveira FJ, Araújo MF, Coelho IC. Comparison between two decades of prevalence of intestinal parasitic diseases and risk factors in a brazilian urban centre. Interdiscip Perspect Infect Dis. 2015;2015:1-8. https://doi. org/10.1155/2015/546705

15. Biscegli TS, Romera J, Candido AB, Santos JD, Candido ECA, Binotto AL. Estado nutricional e prevalência de enteroparasitoses em crianças matriculadas em creche. Rev Paul Pediatr. 2009;27(3): 289-95. https://doi.org/10.1590/S0103-05822009000300009

16. Teixeira JC, Heller L, Barreto ML. Giardia duodenalis infection: risk factors for children living in sub-standard settlements in Brazil. Cad Saúde Pública. 2007;23(6):1489-93. https://doi.org/10.1590/ S0102-311X2007000600024

17. Capuano DM, Rocha GDM. Ocorrência de parasitas com potencial zoonótico em fezes de cães coletadas em áreas públicas do município de Ribeirão Preto, SP, Brasil. Rev Bras Epidemiol. 2006;9(1):81-6. https://doi.org/10.1590/S1415-790X2006000100010

18. Sánchez-Ortiz IA, Leite MA. Zoonosis transmission risk factors according to population habits in Ilha Solteira city, Brazil. Rev Salud Pública. 2011;13(3):504-13.

19. Almeida CG, Marques SMT, Miquelluti DJ, Quadros RM. Giardíase em crianças e cães do mesmo domicílio e de bairros periféricos de Lages, Santa Catarina. Ciênc Saúde (Porto Alegre). 2010;3(1):9-13. https://doi.org/10.15448/1983-652X.2010.1.5487

20. Pilz EML, Schermann LB. Determinantes biológicos e ambientais no desenvolvimento neuropsicomotor em uma amostra de crianças de Canoas/RS. Ciênc Saúde Colet. 2007;12(1):181-90. https://doi. org/10.1590/S1413-81232007000100021
21. Arruda Barbosa LD, Alcântara Sampaio AL, Almeida Melo AL, Nascimento de Macedo AP, Antero Sousa Machado MDF. A educação em saúde como instrumento na Prevenção de parasitoses. Rev Bras Promoção Saúde. 2009;22(4):272-7. https://doi.org/ 10.5020/18061230.2009.p272

22. Gonçalves ALR, Belizário TL, Pimentel JDB, Penatti MPA, Pedroso RDS. Prevalence of intestinal parasites in preschool children in the region of Uberlândia, State of Minas Gerais, Brazil. Rev Soc Bras Med Trop. 2011;44(2):191-3. https://doi.org/10.1590/S003786822011005000022

23. Fonseca EOL, Teixeira MG, Barreto ML, Carmo EH, Costa MDCN. Prevalence and factors associated with geohelminth infections in children living in municipalities with low HDI in North and Northeast Brazil. Cad Saude Publica. 2010;26(1):143-52. https:// doi.org/10.1590/S0102-311X2010000100015

24. Newburg DS, Walker WA. Protection of the neonate by the innate immune system of developing gut and of human milk. Pediatr Res. 2007;61(1):2-8. https://doi.org/10.1203/01.pdr. 0000250274.68571 .18

25. Toma TS, Rea MF. Benefícios da amamentação para a saúde da mulher e da criança: um ensaio sobre as evidências. Cad Saude Publica. 2008;24:S235-46. https://doi.org/10.1590/S0102311X2008001400009

26. Santos Antunes L, Antunes LAA, Corvino MPF, Maia LC. Amamentação natural como fonte de prevenção em saúde. Ciênc Saúde Colet. 2008;13(1):103-9. https://doi.org/10.1590/S141381232008000100015

27. Cacho NT, Lawrence RM. Innate Immunity and Breast Milk. Front Immunol. 2017;29:8:584-94. https://doi.org/10.3389/ fimmu.2017.00584

28. Ballard O, Morrow AL. Human milk composition: nutrients and bioactive factors. Pediatr Clin North Am. 2013;60(1):49-74. https:// doi.org/10.1016/j.pcl.2012.10.002

29. Vesoulis ZA, Mathur AM. Cerebral Autoregulation, Brain Injury, and the Transitioning Premature Infant. Front Pediatr. 2017;5:64. https:// doi.org/10.3389/fped.2017.00064 